\title{
The Feynman effective classical potential in the Schrödinger formulation.
}

\author{
Rafael Ramírez, Telesforo López-Ciudad, and José C. Noya \\ Instituto de Ciencia de Materiales, Consejo Superior de Investigaciones Científicas (C.S.I.C.), \\ Cantoblanco, 28049 Madrid, Spain
}

(November 20, 2016)

\begin{abstract}
New physical insight into the correspondence between path integral concepts and the Schrödinger formulation is gained by the analysis of the effective classical potential, that is defined within the Feynman path integral formulation of statistical mechanics. This potential is related to the quasi-static response of the equilibrium system to an external force. These findings allow for a comprehensive formulation of dynamical approximations based on this potential.
\end{abstract}

PACS numbers: 05.30.-d, 03.65.Ca, 82.20.Wt, 82.20.Db 
The path integral formulation of statistical mechanics is a powerful method to study quantum many-body systems. An essential property of this approach is the mapping of a quantum system onto a classical model of harmonic ring polymers, whose equilibrium properties can be studied with high accuracy. [1] However, dynamical properties at finite temperatures can not be derived with the same sort of rigor, as the solution of the real time path integrals involves complex valued functionals without a suitable sampling function for stochastic integration.

The effective classical potential (ECP), [2] that was introduced by Feynman to study systems in thermodynamic equilibrium, [3] has been a central quantity to derive two quantum dynamical approximations, the quantum transition state theory (QTST), 四 that aims at calculating rate constants of activated processes, and the centroid molecular dynamics (CMD), [5] that aims at calculating real time correlation functions for quantum particles. The definition of the ECP is based on the concept of path centroid, that is the center of gravity of each of the ring polymers that represent the quantum particle. However, in spite of the extensive use of the path centroid in condensed matter and chemical physics studies, its relation to a measurable physical observable and hence the physical meaning of the derived dynamical approximations remain largely unexplained.

In this work, we aim at a deeper understanding of the equivalence between the path integral and the Schrödinger formulation by showing that the centroid coordinate is related to the quasi-static response of the system to an external force. The Schrödinger formulation provides new physical insight into the dynamical approximations based on the ECP. At zero temperature, the ECP is the mean energy of minimum energy wave packets (MEWP's, to be defined below) and the CMD is an approximate dynamics based on these wave packets. Technical details of the analysis are left for a later work. [6]

We begin by reviewing the definition of the ECP in the path integral formulation. The simple case of a quantum particle of mass $m$ having bound states in a one-dimensional potential $V(x)$ is considered. The Hamiltonian of the particle is $H_{0}$. The extension to the many-particle case is, for distinguishable particles, straightforward. [6] At a given temper- 
ature the equilibrium properties are derived from the partition function, $Z_{0}$, and from the particle's probability density, $\rho(x)$. Both are related by the expression $Z_{0}=\int_{-\infty}^{\infty} d x \rho(x)$. The path integral formulation of $\rho(x)$ is [3]

$$
\rho(x)=\int_{x=x(0)}^{x=x(\beta \hbar)} D[x(u)] \exp \left(-\frac{S[x(u)]}{\hbar}\right),
$$

where $S[x(u)]$ is the functional of the Euclidean action of the path $x(u), u$ is the imaginary time that varies between 0 and $\beta \hbar$, and $\beta$ is the inverse temperature $\left(k_{B} T\right)^{-1}$. The paths $x(u)$ can be considered as alternative ways for the propagation of the particle, and the sum over paths can be analyzed in many different ways depending on the different classes into which the alternatives can be divided. [3] The way conducting to the ECP uses the centroid or average point, $x_{c}$, of the path $x(u)$

$$
x_{c}=\frac{1}{\beta \hbar} \int_{0}^{\beta \hbar} d u x(u)
$$

A class of paths is the subset of paths that have the same centroid. A constrained path integral over the class of paths with centroid at $X$ is defined by introducing a delta function in the integrand of Eq. (四),

$$
\rho_{X}(x)=\int_{x}^{x} D[x(u)] \delta\left(X-x_{c}\right) \exp \left(-\frac{S[x(u)]}{\hbar}\right) .
$$

$\rho_{X}(x)$ may be considered as a probability density around the centroid position $X$. As illustration, the normalized function $Z(X)^{-1} \rho_{X}(x)$ obtained by a Monte Carlo path integral simulation of a particle of mass $m=16$ au in a double-well potential, $V_{d p}(x)=\frac{1}{4}\left(x^{2}-1\right)^{2}$, is represented in Fig. 11. The probability density is shown for four values of the centroid coordinate $X$. We also show the probability density of the ground state of the potential $V_{d p}(x)-f x$, for several values of the parameter $f$, that represents an external force acting on the particle. The identity between these ground state probability densities and $Z(X)^{-1} \rho_{X}(x)$ will be explained below. The normalization constant $Z(X)$ is an important quantity

$$
Z(X)=\int_{-\infty}^{\infty} d x \rho_{X}(x)
$$


which has the physical meaning of a probability density for the class of paths with centroid at $X$ and it is called the centroid density. [0] The partition function can be recovered by a sum over all classes, i.e.,

$$
Z_{0}=\int_{-\infty}^{\infty} d X Z(X)
$$

This integral, after substitution of $Z(X)$ by the following definition,

$$
Z(X)=\left(\frac{m}{2 \pi \beta \hbar^{2}}\right)^{1 / 2} \exp \left[-\beta F_{e f}(X)\right]
$$

has the same form as the partition function of a classical particle moving in the potential, $F_{\text {ef }}(X)$, which is the ECP. We summarize some properties of the ECP: [2,7] i) all the thermodynamic properties that depend on $Z_{0}$ can be derived from it; ii) it is temperature dependent; iii) its calculation is analytical only for quadratic potentials, but variational approximations are available; iv) its high temperature limit is the actual potential $V(x)$.

The centroid density $Z(X)$ (or the ECP) and the probability densities $Z(X)^{-1} \rho_{X}(x)$ are important quantities in the theory of path integrals, whose correspondence to the Schrödinger formulation has not been clearly stated. In the following, we show how the centroid density is related to a physical observable. We first consider a Hamiltonian depending on an external force $f$ acting on the particle, $H_{f}=H_{0}-f x$. The path integral representation of the partition function $Z_{f}$, corresponding to the Hamiltonian $H_{f}$, can be expressed as 8

$$
Z_{f}=\int_{-\infty}^{\infty} d X Z(X) e^{\beta f X}=Z_{0}\left\langle e^{\beta f X}\right\rangle
$$

where $Z(X)$ is the centroid density for the particle with Hamiltonian $H_{0}$. The angle brackets show an average over the normalized centroid density. If $Z(X)$ is known, the partition function $Z_{f}$ can be derived by Eq. (7) for any arbitrary value of the external force $f$. Thus, the analysis of classes of paths with fixed centroid allows to derive the thermodynamic properties of a whole family of systems whose Hamiltonian depends on a parameter $f$. The moments of the centroid density are defined as 


$$
\left\langle X^{n}\right\rangle=Z_{0}^{-1} \int_{-\infty}^{\infty} d X Z(X) X^{n} .
$$

The physical meaning of Eq. (77) is that the ratio of partition functions $Z_{f} / Z_{0}$ is the function generating the moments of the centroid density. The moments are derived by differentiation of $Z_{f} / Z_{0}$ with respect to the variable $\beta f$. The result is simpler if we use the free energy, $F_{f}$, defined as $\exp \left(-\beta F_{f}\right)=Z_{f}$. The first two moments are obtained as

$$
\begin{gathered}
\langle X\rangle=-\left(\frac{\partial F_{f}}{\partial f}\right)_{f=0}, \\
\left\langle X^{2}\right\rangle-\langle X\rangle^{2}=-k_{B} T\left(\frac{\partial^{2} F_{f}}{\partial f^{2}}\right)_{f=0} .
\end{gathered}
$$

The mean-squared deviation of $X$ is often called the classical delocalization of the particle. Higher order derivatives of $F_{f}$ lead to higher moments of the centroid density. [6] If the moments are known, then the centroid density itself will be fully determined. We have then a formal relation between the centroid density and the change in the free energy as a function of a quasi-static force acting on the particle. This central result reveals the physical meaning of the centroid density within the Schrödinger formulation.

This correspondence between the centroid density and the Schrödinger formulation is valid for arbitrary temperatures. The zero temperature limit is particularly interesting, as quantum effects are then most important. Before analyzing this limit, it is convenient to study a property of the Hamiltonian $H_{f}$ that leads to the definition of MEWP's. We look for quantum states of the particle whose mean energy, $E_{0}^{\min }=\left\langle\psi_{f}\left|H_{0}\right| \psi_{f}\right\rangle$, is minimum against small variations of $\left|\psi_{f}\right\rangle$. Moreover, the states must satisfy two constraints: i) their mean position is fixed at an arbitrary value $\bar{x}=\left\langle\psi_{f}|x| \psi_{f}\right\rangle$; ii) they are normalized $\left\langle\psi_{f} \mid \psi_{f}\right\rangle=1$. By straightforward application of calculus of variations, [6] one finds that $\left|\psi_{f}\right\rangle$ is the ground state of $H_{f}$

$$
\left(H_{0}-f x\right)\left|\psi_{f}\right\rangle=E_{f}\left|\psi_{f}\right\rangle,
$$

$f$ and $E_{f}$ are Lagrange multipliers chosen so that the constraints $i$ and $i i$ are satisfied. Note 
that $f$ is an implicit function of the arbitrary position $\bar{x}$, i.e., $f \equiv f(\bar{x})$. The minimum energy, $E_{0}^{\min }$, as a function of $\bar{x}$, is derived from Eq. (11]) as

$$
E_{0}^{\min }(\bar{x})=E_{f}+f \bar{x} .
$$

We call the states $\left|\psi_{f}\right\rangle$ the MEWP's of the potential $V(x)$ whose mean energy is $E_{0}^{\min }(\bar{x})$. We show next how these states are related to the ECP at zero temperature.

At $T=0$, the free energy is equal to the ground state energy, $F_{f}=E_{f}$, and from Eqs. (9), and (10) we obtain

$$
\langle X\rangle=-\left(\frac{\partial E_{f}}{\partial f}\right)_{f=0} ;\left\langle X^{2}\right\rangle-\langle X\rangle^{2}=0 .
$$

$\langle X\rangle$ is then the expectation value of the derivative of the Hamiltonian operator in Eq. (11) with respect to $f$ at $f=0$, i.e., $\langle X\rangle$ is equal to the mean position of the ground state of the Hamiltonian $H_{0}$

$$
\langle X\rangle=\left\langle\psi_{0}|x| \psi_{0}\right\rangle=\bar{x}_{0}
$$

The mean-squared deviation of the centroid density vanishes [Eq. (13], thus the centroid density must be a delta function centered at $\bar{x}_{0}$. This result implies that only the class of paths with centroid at $X \equiv \bar{x}_{0}$ contributes to the path integral in Eq. (1), i.e.,

$$
\lim _{T \rightarrow 0} Z_{0}^{-1} \rho(x)=\lim _{T \rightarrow 0} Z(X)^{-1} \rho_{X}(x), \text { for } X \equiv \bar{x}_{0}
$$

This identity provides a clear physical interpretation of path integral quantities at $T=0$. It implies that the centroid density and the partition function of the particle are related by: $Z(X)=Z_{0} \delta\left(X-\bar{x}_{0}\right)$. Moreover, the asymptotic behavior of $Z_{0}$ and $Z\left(\bar{x}_{0}\right)$ as $T \rightarrow 0$ is given by exponential functions of the ground state energy, $\exp \left(-\beta E_{0}\right)$, and the ECP, $\exp \left[-\beta F_{e f}\left(\bar{x}_{0}\right)\right]$, respectively. Then, $F_{e f}\left(\bar{x}_{0}\right)$ must be equal to the ground state energy. This result is a particular case of a relation valid for any arbitrary centroid position $X$. An essential step for this generalization is that the probability densities $Z(X)^{-1} \rho_{X}(x)$ are invariant under any change in the Hamiltonian $H_{0}$ that is linear in the coordinate $x$. The 
final result of this generalization is: [6] i) at $T=0$ the normalized probability density for a given class of paths, $Z(X)^{-1} \rho_{X}(x)$, is identical to the probability density of the MEWP, $\left|\left\langle x \mid \psi_{f}\right\rangle\right|^{2}$, whose mean position is at $\bar{x} \equiv X$; ii) at $T=0$ the $E C P, F_{\text {ef }}(X)$, is equal to the mean energy, $E_{0}^{\min }(\bar{x})$ of the MEWP whose mean position is at $\bar{x} \equiv X$.

In Fig. [1 we showed the probability density $Z(X)^{-1} \rho_{X}(x)$ obtained from Monte Carlo path integral simulations of a particle in a double-well potential at temperature $k_{B} T=10^{-3}$ au. This value is a small fraction of the lowest excitation energy, $\Delta E_{0}=29 \times 10^{-3}$ au (tunnel splitting), and therefore a good approximation to the zero temperature limit. For comparison, the probability density of MEWP's, obtained numerically as the ground state of the potential $V_{d p}(x)-f x$, are shown by broken lines. The identity of both probability densities is an important result that clarifies the physical meaning of fixed centroid path integrals. In Fig. 2 the energies $V_{d p}(\bar{x})$ and $E_{0}^{\min }(\bar{x})$ are shown. These curves correspond to the limits of infinite and zero temperature for the ECP, respectively.

In QTST the second derivative of the ECP, $F_{e f}(X)$ with respect to $X$, has been shown to be an important quantity to determine the pre-exponential factor of rate constants. [4] If the effect of a small external force on a stationary quantum state is approximated by a rigid spatial displacement of the state, it can be shown [6] that in the zero temperature limit the second derivative of the ECP with respect to $X$ gives an approximation to the first excitation energy of the Hamiltonian $H_{f}$, i.e.

$$
\hbar\left[\frac{1}{m}\left(\frac{\partial^{2} E_{0}^{\min }(\bar{x})}{\partial \bar{x}^{2}}\right)\right]^{\frac{1}{2}} \simeq \Delta E_{f} .
$$

In Fig. 3 we display the exact values of $\Delta E_{f}$ as a function of $\bar{x}$ (remember that $f$ is an implicit function of $\bar{x}$ ). The l.h.s of Eq.(16), drawn by a broken line, was obtained by numerical differentiation of the function $E_{0}^{\min }(\bar{x})$, shown in Fig. 2. At $\bar{x}=0$ the approximation overestimates the energy of the tunnel splitting, $\Delta E_{0}$, by $25 \%$.

An approximation to the time evolution of the particle can be formulated with MEWP's: i) only MEWP's with mean position $\bar{x}$ and momentum $\bar{p}$ are allowed as time dependent states, i.e., these are of the form $\left\langle x \mid \psi_{f}\right\rangle \exp (i \bar{p} x / \hbar)$. The value of the mean force is $\bar{f}=-f$; 
ii) the time dependence of $\bar{x}$ and $\bar{p}$ is given by the Ehrenfest relations

$$
\frac{d \bar{x}}{d t}=\frac{\bar{p}}{m} ; \frac{d \bar{p}}{d t}=\bar{f} .
$$

The total energy of the MEWP, $E_{0}^{\min }(\bar{x})+\bar{p}^{2} /(2 m)$, is conserved along this time evolution. This approximation, formulated without any reference to path integral concepts, can be recognized as the zero temperature limit of CMD. The formulation of CMD as an approximate dynamics based on MEWP's provides new physical insight into this approximation. In Fig. 国 we show a phase space representation of the time evolution of $\bar{x}(t)$ and $\bar{p}(t)$ for an initial MEWP with $\bar{x}(0)=-0.7$ au and $\bar{p}(0)=0$ moving in the potential $V_{d p}$. The exact trajectory was obtained by numerical solution of the time dependent Schödinger equation. The CMD trajectory, derived from Eq. (17), is that of a classical particle moving in the ECP shown in Fig. 2. For comparison the phase space trajectory of a classical particle in the potential $V_{d p}$ is also shown. The CMD result resembles the real time trajectory.

Summarizing, the centroid density has been derived in the Schrödinger formulation with the help of a function generating its moments. The centroid density is related to the response of the system to a quasi-static force. For potentials with bound states the centroid density converges to a delta function as $T \rightarrow 0$. Several results have been found in this limit: $i$ ) the ECP is the mean energy of the MEWP's of the potential; ii) the second derivative of the ECP with respect to the centroid position approximates the first excitation energy of the system; iii) CMD is an approximate dynamics based on MEWP's.

This work was supported by DGICYT (Spain) under contract PB96-0874. We thank E. Artacho and J.J. Sáenz for helpful discussions. 


\section{REFERENCES}

[1] D. M. Ceperley, Rev. Mod. Phys. 67, 279 (1995).

[2] For a review see: A. Cuccoli, R. Giachetti, V. Tognetti, R. Vaia, and P. Verrucchi, J. Phys.: Condens. Matter 7, 7891 (1995).

[3] R.P. Feynman and A.R. Hibbs, Quantum Mechanics and Path Integrals (McGraw-Hill, New York, 1965).

[4] M. J. Gillan, Phys. Rev. Lett. 58, 563 (1987), and references therein.

[5] J. Cao and G. A. Voth, J. Chem. Phys. 100, 5106 (1994).

[6] R. Ramírez, and T. López-Ciudad, to be published.

[7] D. Acocella, G.K. Horton, and E.R. Cowley, Phys. Rev. Lett. 74, 4887 (1995).

[8] H. Kleinert, Pfadintegrale (Wissenschaftverlag, Mannheim, 1993) p. 254. 


\section{FIGURES}

FIG. 1. The continuous lines show normalized probability densities, $Z(X)^{-1} \rho_{X}(x)$, obtained by fixed centroid path integral simulations at $k_{B} T=10^{-3}$ au, in the potential $V_{d p}(x)$. The dotted lines (on top of the continuous ones) are the probability densities of the ground state of the potential $V_{d p}(x)-f x$, that is shown by broken lines for several values of $f$.

FIG. 2. The ECP in the limit $T \rightarrow 0$ (broken line) and the double well potential $V_{d p}$ (continuous line).

FIG. 3. First excitation energy $\Delta E_{f}$, for the Hamiltonian $H_{f}$ (continuous line) as a function of the mean position $\bar{x}$ of the ground state of $H_{f}$, and its approximation based on the second derivative of the ECP (broken line).

FIG. 4. Phase space trajectory showing the time evolution of the mean position and momentum of an initial state corresponding to a MEWP of the $V_{d p}$ potential with $\bar{x}(0)=-0.7$ au and $\bar{p}(0)=0$. The exact result is compared to the CMD approximation and to the classical trajectory. 


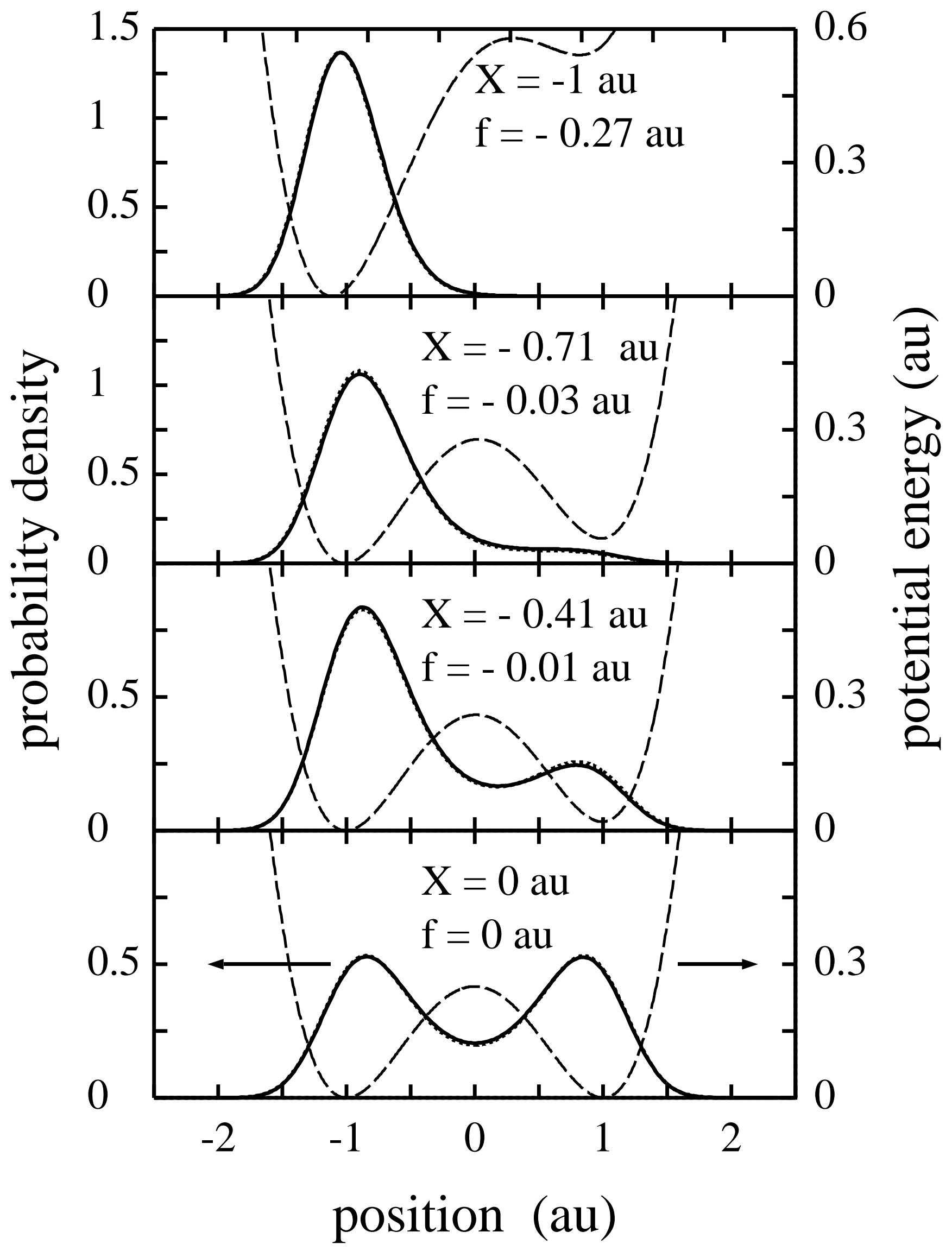




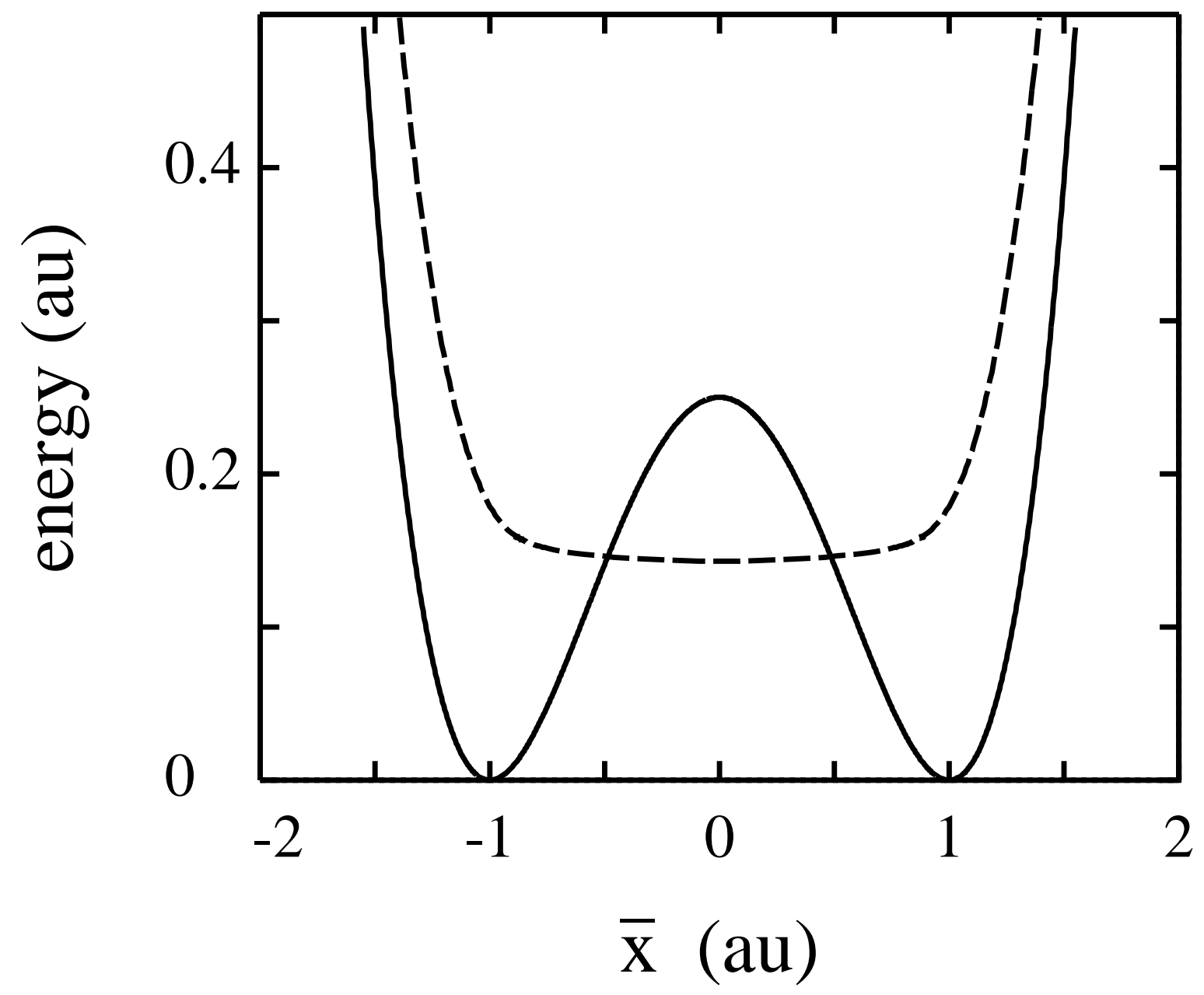




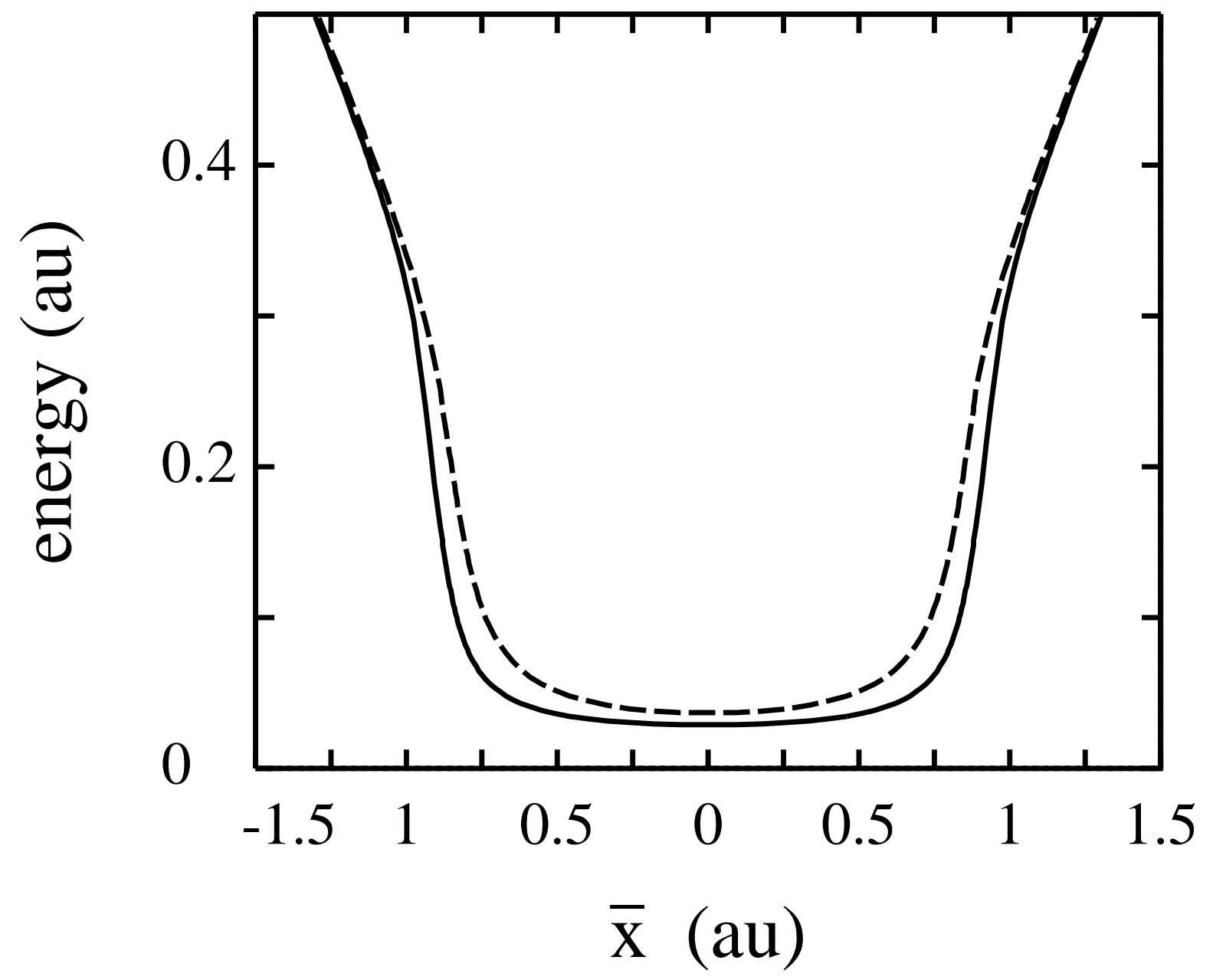




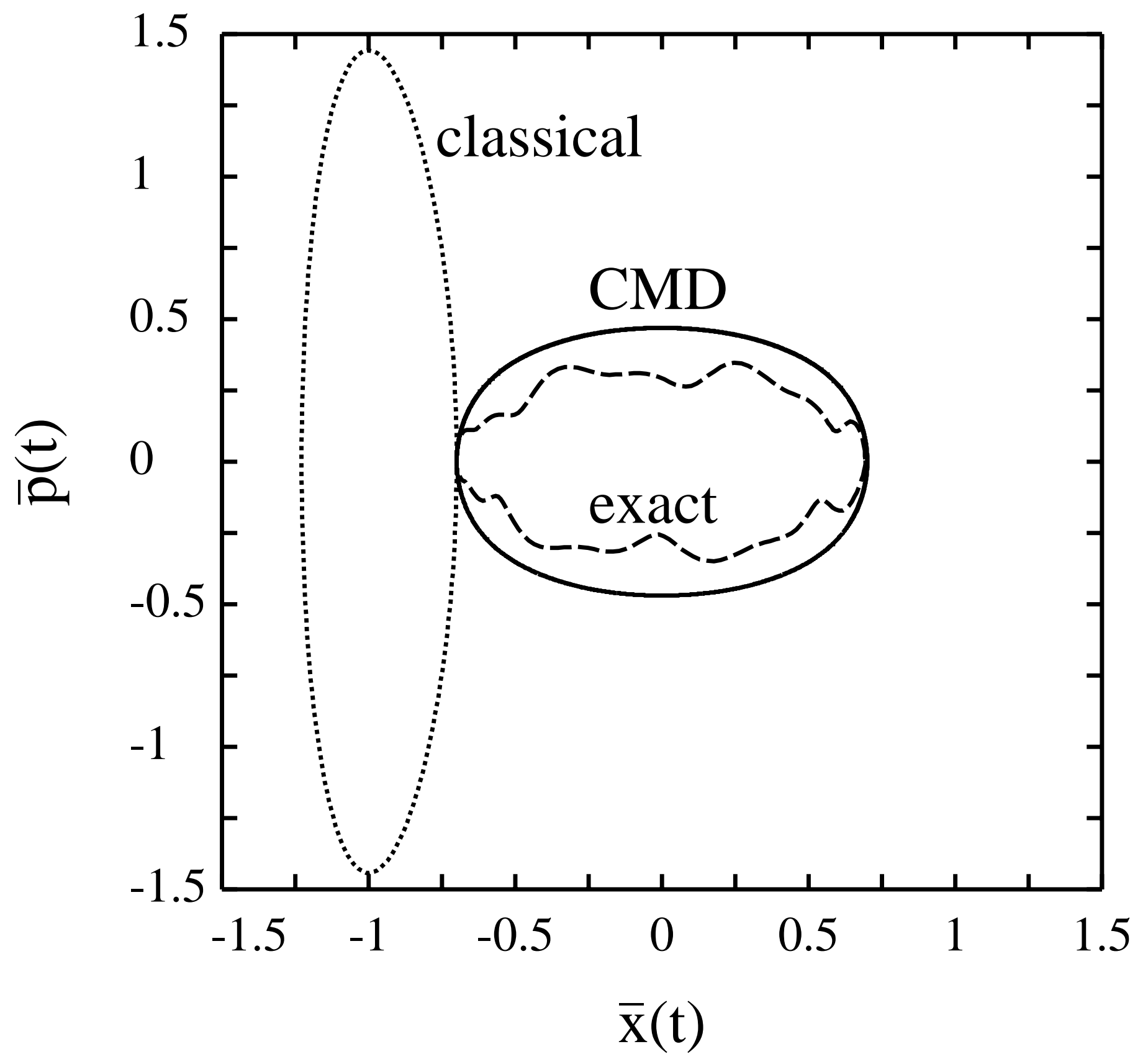

